\title{
Iterative Solution of Dielectric Waveguide Problems via Schur Complement Preconditioners
}

\author{
Tahir Malas ${ }^{1,2}$ and Levent Gürel ${ }^{1,2 *}$ \\ ${ }^{1}$ Department of Electrical and Electronics Engineering \\ ${ }^{2}$ Computational Electromagnetics Research Center (BiLCEM) \\ Bilkent University, TR-06800, Bilkent, Ankara, Turkey \\ E-mail: \{tmalas,lgurel\}@ee.bilkent.edu.tr
}

\section{Introduction}

Surface integral-equation methods accelerated with the multilevel fast multipole algorithm provide suitable mechanisms for the solution of dielectric problems. In particular, recently developed formulations increase the stability of the resulting matrix equations, hence they are more suitable for iterative solutions [1]. Among those formulations, we consider the combined tangential formulation (CTF), which produces more accurate results, and the electric and magnetic current combined-field integral equation (JMCFIE), which produces better-conditioned matrix systems than other formulations $[1,2]$.

CTF and JMCFIE can be regarded as the counterparts of the electric-field integral equation (EFIE) and the combined-field integral equation (CFIE), which are commonly used in perfect-electric-conductor (PEC) problems. For real-life problems with high dielectric constants, however, matrix systems resulting from both CTF and JMCFIE represent a significant challenge in terms of convergence because of indefiniteness and poor spectral properties. To overcome this problem, we propose two variants of Schur complement preconditioners that are improved versions of those introduced in [3]. Then, we present the solutions of two large dielectric perforated waveguide problems using these preconditioners.

\section{Schur Complement Preconditioning}

In order to devise an effective preconditioner for dielectric problems, the $2 \times 2$-partitioned near-field matrix system

$$
\left[\begin{array}{ll}
\overline{\boldsymbol{A}}_{11}^{N F} & \overline{\boldsymbol{A}}_{12}^{N F} \\
\overline{\boldsymbol{A}}_{21}^{N F} & \overline{\boldsymbol{A}}_{22}^{N F}
\end{array}\right] \cdot\left[\begin{array}{l}
\boldsymbol{v}_{1} \\
\boldsymbol{v}_{2}
\end{array}\right]=\left[\begin{array}{l}
\boldsymbol{w}_{1} \\
\boldsymbol{w}_{2}
\end{array}\right]
$$

should be efficiently solved with minimum computational requirements. In this context, incomplete-LU factorizations fail to be a proper solution because of the instability of the factors and the high memory requirement of this approach [4]. On the other hand, it is possible to provide fast and yet successful approximations to solutions of such partitioned matrix systems using Schur complement reduction. With this method, the solution of the $2 N \times 2 N$ near-field system in (1) can be reduced into the solutions of $N \times N$ reduced systems

$$
\overline{\boldsymbol{S}} \cdot \boldsymbol{v}_{2}=\boldsymbol{w}_{2}-\overline{\boldsymbol{A}}_{21}^{N F} \cdot\left(\overline{\boldsymbol{A}}_{11}^{N F}\right)^{-1} \cdot \boldsymbol{w}_{\mathbf{1}}
$$

\footnotetext{
${ }^{\dagger}$ This work was supported by the Scientific and Technical Research Council of Turkey (TUBITAK) under the Research Grant 107E136, by the Turkish Academy of Sciences in the framework of the Young Scientist Award Program (LG/TUBA-GEBIP/2002-1-12), and by contracts from ASELSAN and SSM.
} 
and

$$
\overline{\boldsymbol{A}}_{11}^{N F} \cdot \boldsymbol{v}_{1}=\boldsymbol{w}_{\mathbf{1}}-\overline{\boldsymbol{A}}_{12}^{N F} \cdot \boldsymbol{v}_{2}
$$

where

$$
\overline{\boldsymbol{S}}=\overline{\boldsymbol{A}}_{22}^{N F}-\overline{\boldsymbol{A}}_{21}^{N F} \cdot\left(\overline{\boldsymbol{A}}_{11}^{N F}\right)^{-1} \cdot \overline{\boldsymbol{A}}_{12}^{N F}
$$

is the Schur complement. Approximate solutions to (2) and (3), which can be obtained either directly or iteratively, induce effective preconditioners. The preconditioner that uses the direct approach is called "the approximate Schur preconditioner (ASP)" and the one that uses the iterative approach "the iterative Schur preconditioner (ISP)." When the number of inner iterations for the reduced systems are not fixed, the outer iterative solver employed for ISP should be chosen as a flexible Krylov solver [5].

Both ASP and ISP require approximate inverses of $\overline{\boldsymbol{A}}_{11}^{N F}$ and $\overline{\boldsymbol{S}}$. For ISP, approximate inverses should be used as "inner" preconditioners to accelerate iterative solutions of the reduced systems (2) and (3). For $\overline{\boldsymbol{A}}_{11}^{N F}$, we use a sparse approximate inverse (SAI) based on Frobenius-norm minimization, which has proven to yield a successful approach in PEC problems formulated with EFIE or CFIE [6]. This SAI, which we denote by $\overline{\boldsymbol{M}}_{11}$, uses the sparsity pattern of $\overline{\boldsymbol{A}}_{11}^{N F}$. Hence, it costs only one-fourth of the memory consumed by the near-field matrix. $\overline{\boldsymbol{M}}_{11}$ is also used to approximate the inverse of $\overline{\boldsymbol{A}}_{11}^{N F}$ in the right-hand side of (2) and in matrix-vector multiplications (MVMs) of $\overline{\boldsymbol{S}}$ for ISP.

In the literature, sparse approximations to both $\bar{S}$ itself and the inverse of $\bar{S}$ have been developed when the $(1,1)$ partition is zero or it has a small size [5], which are not the cases for CTF or JMCFIE. As proposed in [3], we can approximate the inverse of $\overline{\boldsymbol{S}}$ by the SAI of $\overline{\boldsymbol{A}}_{11}^{N F}$, which is equal to $\overline{\boldsymbol{M}}_{11}$ due to identical diagonal partitions of CTF or JMCFIE matrices. However, this choice fails to provide a successful approximate inverse for a large dielectric constant, since the near-field matrix loses its diagonal dominance [1]. To include the second term of $\overline{\boldsymbol{S}}$, only diagonal blocks of the partitions can be taken into account, as in [2]. However, this approach also fails in CTF and for large dielectric constants in JMCFIE.

In this paper, we propose a more effective approach. To retain the near-field matrix information beyond the diagonal partitions and the information in the second term of $\overline{\boldsymbol{S}}$, we first compute a sparse approximation to $\overline{\boldsymbol{S}}$ in the form of

$$
\widetilde{\overline{\boldsymbol{S}}}=\overline{\boldsymbol{A}}_{22}^{N F}-\overline{\boldsymbol{A}}_{21}^{N F} \odot \overline{\boldsymbol{M}}_{11} \odot \overline{\boldsymbol{A}}_{12},
$$

where $\odot$ denotes an incomplete matrix-matrix multiplication obtained by retaining the nearfield sparsity pattern. Then, the inverse of $\overline{\boldsymbol{S}}$ is approximated by

$$
\overline{\boldsymbol{M}}_{S} \approx \widetilde{\overline{\boldsymbol{S}}}^{-1} \approx \overline{\boldsymbol{S}}^{-1}
$$

where $\overline{\boldsymbol{M}}_{S}$ denotes a SAI of $\widetilde{\overline{\boldsymbol{S}}}$. If the entries of the near-field partitions are stored rowwise, the incomplete matrix-matrix multiplications in (5) can be performed in $\mathcal{O}(N)$ time using the $i k j$ loop order of the matrix-matrix multiplication [7]. The proposed SAI for $\bar{S}$ generates a successful approximation of the inverse of $\bar{S}$ that can be used as a direct solver or as a strong preconditioner. 
Table 1: Solutions of the waveguide problems with Schur complement preconditioners.

\begin{tabular}{|c|c|c|c|c|c|c|c|}
\hline \multicolumn{3}{|c|}{ CTF } & \multicolumn{2}{|c|}{ Setup } & \multicolumn{3}{|c|}{ ASP } \\
\hline Size $(\mathrm{cm})$ & Unknowns & Level & $\overline{\bar{M}}_{11}$ & $\overline{\bar{M}}_{S}$ & Iter & Solution & Total \\
\hline $0.6 \times 26 \times 34$ & 475,782 & 7 & 4.4 & 4.6 & 697 & 19.0 & 28.0 \\
\hline $0.6 \times 29 \times 38$ & 597,462 & 7 & 5.7 & 6.1 & 829 & 27.6 & 39.4 \\
\hline \multicolumn{3}{|c|}{$\begin{array}{l}\text { JMCFIE } \\
\end{array}$} & \multicolumn{2}{|c|}{ Setup } & \multicolumn{3}{|c|}{ ISP } \\
\hline Size $(\mathrm{cm})$ & Unknowns & Level & $\overline{\boldsymbol{M}}_{11}$ & $\overline{\bar{M}}_{S}$ & Iter & Solution & Total \\
\hline $0.6 \times 26 \times 34$ & 475,782 & 7 & 4.4 & 4.6 & 110 & 6.3 & 15.4 \\
\hline $0.6 \times 29 \times 38$ & 597,462 & 7 & 5.7 & 6.1 & 139 & 10.0 & 21.8 \\
\hline
\end{tabular}

\section{Results}

We show the effectiveness of ASP and ISP on a perforated waveguide problem, for which the relative permittivity is 12.0 [8]. The problem is investigated at its resonance frequency, i.e., $7.6 \mathrm{GHz}$, where the transmission takes place most efficiently. Because of the tiny air holes, we use a fine mesh with $\lambda / 20$ triangles. The generalized-minimal-residual (GMRES) method without a restart or its flexible version is used as the iterative solver [9]. Iterations are started with a zero initial guess and terminated when the relative residual error is reduced by $10^{-3}$ or at a maximum of 1,000 iterations.

In Table 1, we show the solutions of two instances of the waveguide problem. We first note that neither of these problems can be solved with the block-diagonal preconditioners described in [2] or with the ISP proposed in [3], which uses the preconditioner $\overline{\boldsymbol{M}}_{11}$ for both $\overline{\boldsymbol{A}}_{11}^{N F}$ and $\overline{\boldsymbol{S}}$. Because of the fine mesh of the problem, the near-field matrices are denser than usual, hence, the application cost of ISP is significantly larger than ASP. As a result, the minimum solution times are obtained with ASP for CTF. For JMCFIE, on the other hand, the number of iterations can be drastically reduced with ISP, which compensates its extra cost.

To compare the solutions of the two formulations in terms of accuracy, we present in Fig. 1 the near-zone electric fields for the larger problem in Table 1. The total field is calculated point-wise inside and outside the problem. We observe that there is a high discrepancy between the CTF and JMCFIE solutions for this problem. As mentioned in [1], this difference is related to the deteriorating accuracy of JMCFIE for complicated structures which have high dielectric constants.

\section{Concluding Remarks}

In this paper, we consider solutions of a real-life dielectric problem using CTF and JMCFIE formulations. We offer two novel preconditioners, namely ASP and ISP, to overcome the convergence problem. The matrix equation obtained from JMCFIE benefits more from preconditioning and it is solved faster than the one obtained from CTF. However, a comparison of the near-zone fields shows that solutions obtained with JMCFIE may not be reliable for the analysis of real-life problems that involve complex shapes and high dielectric constants. 


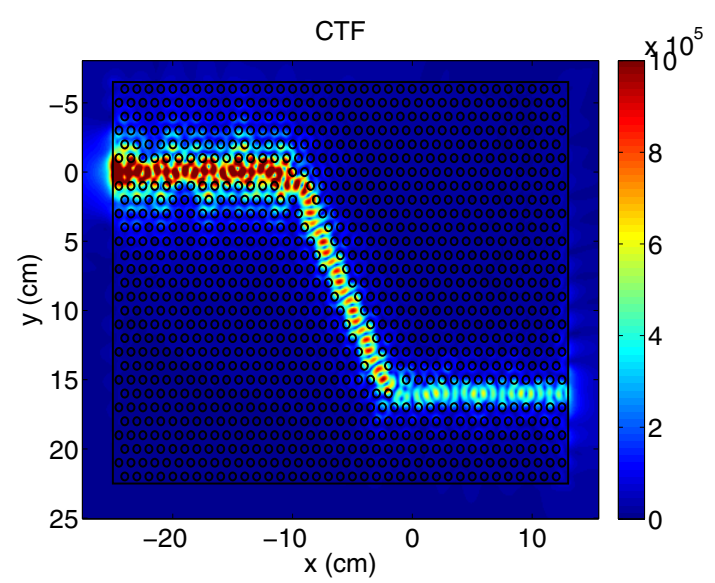

(a)

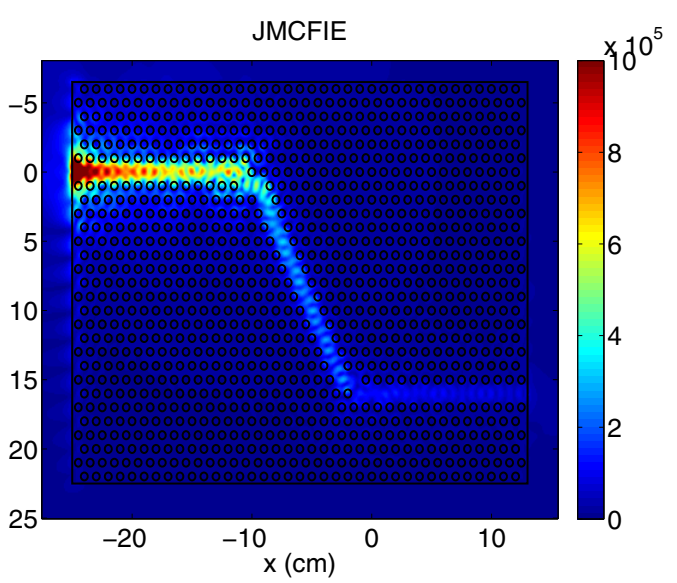

(b)

Figure 1: Near-zone electric fields of the problem when illuminated by a Hertzian dipole.

\section{References}

[1] P. Ylä-Oijala, M. Taskinen, and S. Järvenpää, "Analysis of surface integral equations in electromagnetic scattering and radiation problems," Eng. Anal. Boundary Elem., vol. 32, no. 3, pp. 196-209, 2008.

[2] Ö. Ergül and L. Gürel, "Comparison of integral-equation formulations for the fast and accurate solution of scattering problems involving dielectric objects with multilevel fast multipole algorithm," IEEE Trans. Antennas Propagat., vol. 57, no. 1, pp. 176-187, Jan. 2009.

[3] T. Malas and L. Gürel, "An effective preconditioner based on the Schur complement reduction for integral-equation formulations of dielectric problems," in IEEE International Symposium on Antennas and Propagation, Charleston, South Carolina, USA, June 2009.

[4] — " "Increasing robustness and efficiency of surface-integral-equation solutions of dielectric problems with approximate Schur preconditioners," in 2009 Computational Electromagnetics International Workshop (CEM'09), İzmir, Turkey, July 2009.

[5] M. Benzi, G. H. Golub, and J. Liesen, "Numerical solution of saddle point problems," Acta Numer., vol. 14, pp. 1-137, 2005.

[6] T. Malas and L. Gürel, "Accelerating the multilevel fast multipole algorithm with the sparse-approximate-inverse (SAI) preconditioning," SIAM J. Sci. Comput., vol. 31, no. 3, pp. 1968-1984, 2009.

[7] G. H. Golub and C. F. van Loan, Matrix Computations. Johns Hopkins University Press, 1996.

[8] S. G. Johnson, S. Fan, P. R. Villeneuve, and J. D. Joannopoulos, "Guided modes in photonic crystal slabs," Physical Review B, vol. 60, no. 8, pp. 5751-5758, 1999.

[9] Y. Saad, Iterative Methods for Sparse Linear Systems, 2nd ed. Philadelphia, PA, USA: SIAM, 2003. 